C2010 IEEE. Personal use of this material is permitted. However, permission to reprint/republish this material for advertising or promotional purposes or for creating new collective works for resale or redistribution to servers or lists, or to reuse any copyrighted component of this work in other works must be obtained from the IEEE 


\title{
Segmentation of Pulmonary Nodules in Thoracic CT Scans: A Region Growing Approach
}

\author{
Jamshid Dehmeshki*, Member, IEEE, Hamdan Amin, Manlio Valdivieso, and Xujiong Ye
}

\begin{abstract}
This paper presents an efficient algorithm for segmenting different types of pulmonary nodules including high and low contrast nodules, nodules with vasculature attachment, and nodules in the close vicinity of the lung wall or diaphragm. The algorithm performs an adaptive sphericity oriented contrast region growing on the fuzzy connectivity map of the object of interest. This region growing is operated within a volumetric mask which is created by first applying a local adaptive segmentation algorithm that identifies foreground and background regions within a certain window size. The foreground objects are then filled to remove any holes, and a spatial connectivity map is generated to create a 3-D mask. The mask is then enlarged to contain the background while excluding unwanted foreground regions. Apart from generating a confined search volume, the mask is also used to estimate the parameters for the subsequent region growing, as well as for repositioning the seed point in order to ensure reproducibility. The method was run on 815 pulmonary nodules. By using randomly placed seed points, the approach was shown to be fully reproducible. As for acceptability, the segmentation results were visually inspected by a qualified radiologist to search for any gross misssegmentation. $84 \%$ of the first results of the segmentation were accepted by the radiologist while for the remaining $16 \%$ nodules, alternative segmentation solutions that were provided by the method were selected.
\end{abstract}

Index Terms-Fuzzy connectivity, local adaptive segmentation, nodule segmentation, region growing.

\section{INTRODUCTION}

$\mathbf{T}$ HE mortality rate for lung cancer is higher than that of other kinds of cancers around the world. To improve the chance of survival, an early detection of suspicious lesions of cancer is crucial.

Lung nodule detection is a challenging task in medical imaging as lung nodules may be difficult to detect using computed tomography (CT) scans due to low contrast, small size, or the location of the nodule within an area of complicated anatomy. For this reason, several methods for developing computer aided detection (CAD) methods of nodules have been reported in the literature to assist radiologists [1]-[6]. The volume analysis of lung nodules is also important for diagnosis.

Manuscript received July 25, 2007; revised August 21, 2007. Asterisk indicates corresponding author.

*J. Dehmeshki is with the Faculty of Computing, Information Systems and Mathematics, Kingston University, Kingston Upon Thames KT1 2EE, U.K. (e-mail: j.dehmeshki@kingston.ac.uk).

H. Amin, M. Valdivieso, and X. Ye are with the Medicsight Plc, London W1J 5AT, U.K.

Color versions of one or more of the figures in this paper are available online at http://ieeexplore.ieee.org.

Digital Object Identifier 10.1109/TMI.2007.907555
In relation to this, the automatic calculation of nodule volumes [7] has been increasingly used as an accurate and reproducible technique. When compared to manual measurement of axial diameter, the automatic calculation of nodule volume is a more appropriate and sensitive method of recording nodule growth. However, a common problem is that when the nodule is attached to blood vessels or surrounded by a structure with a similar intensity (i.e., X-ray attenuation coefficients), it becomes difficult to detect the extent of a lung nodule purely by inspection. Thus, the detection of the extent of a lung nodule through nodule segmentation is important for volumetric measurement of nodules and an accurate diagnosis. (Note that although the characterization of nodules-using measurements such as volume and diameter-following segmentation is important and, indeed, the ultimate aim of this research, that characterization is not within the scope of this paper.)

An early method of nodule segmentation was described in [8] in which dynamic programming was applied to the each cross section of each nodule separately. This 2-D method relied on manual initialization and is a 2-D approach. Other approaches based on the deformable surface include [9], [10].

One problem with many techniques is that they impose a model which is not applicable for all types of lung nodule. For example, the approaches described in [11] and [12] are more applicable for spherical or ellipsoid nodules, respectively. This is also the case for the methods introduced in [13] and [14] where the volumes of nodules were estimated using the volume of an ellipsoid. The ellipsoidal approximation of a nodule boundary [13] results in a low intraobserver and interobserver variability for volumetric measurement. This is a key issue for followup studies of nodules (comparing a nodule scanned at two different times). However, failing to describe the fine structure of a nodule may result in an inaccurate estimation of its volume, particularly for nodules with irregular surfaces or small nodules with pleural attachments.

A morphology based approach was introduced in [15]. One problem with this method is its sensitivity to the morphology template size which makes it difficult to choose a suitable template size for all different kinds of nodules. Using the morphological approach might remove surface detail of nodules such as fine spiculations. As the authors indicated in [15], this algorithm is targeted for small and high contrast nodules; thus for large nodules, especially when a blood vessel is attached to a nodule from one side, the algorithm might fail to properly delineate the nodules. Similar approaches like this can be found in [16] and [17].

The region growing technique [18]-[24] is yet another approach to nodule segmentation. It usually includes the following steps. 
Step 1) Identifying a seed point (for example, within a nodule candidate).

Step 2) Calculating the connectivity of the points of the images to the seed point.

Step 3) Applying a selection criteria to the connectivity of the points (from Step 2 above) in order to identify the extent of the nodule (Halting criteria).

There are a number of pulmonary nodule types that need to be addressed. Some pulmonary nodules (e.g., calcified nodules) are very dense, having very high contrast which readily separate them from the background, even when they are attached to vasculature. Other nodules may have sufficient contrast to segment them from the background, but also be attached to blood vessels with the same or similar intensity contrast relative to the local background. Further, there are some pulmonary nodules which have a density close to the surrounding lung parenchyma. Fig. 2 shows some examples of different types of pulmonary nodules.

Due to this diversity of nodule types, there is an important and challenging need to design a robust region growing method for lung nodule boundary detection. We ideally require that a region growing method should have the following features, which will be addressed throughout the paper.

1) Seed Point Sensitivity: The detected boundary should not vary when different seed points within the nodule are used. Seed points may be selected by any suitable means for example, manual or automatic selection.

2) Delineation of Nodule Boundary: The algorithm should separate the nodule boundary from surrounding tissue with similar density or X-ray attenuation coefficient such as the blood vessel.

3) Few Number of Input Parameters and Use of Adaptive Parameter Settings: Ideally, methods should not require any input parameters; where required, they should preferably be as few as possible. If the method requires parameters to be set, then some means of adaptively or automatically estimating those values should be incorporated. This should preferably be on a per nodule basis to rigorously deal with the variety of nodule types.

4) Halting Criteria: To get the region growing algorithm to stop at the desired boundary, region growing methods should not use predefined threshold values. Where required, feature 3 above should be applied.

5) Morphology: The region growing method should make use of contextual information. For example, if the apparent shape of part of a nodule indicates a sphere, then this evidence should have an influence on the dynamic path of growth of the region.

6) Usability: An effective implementation of region growing methods into the radiology workflow is essential. User interactivity should be minimal and the method should be relatively fast. It is also useful to provide multiple segmentation results for review by the user. For example, where the mathematically optimum result is not to the user's satisfaction (such as failure in separating a nodule from the blood vessel), the user should be allowed to select one of the other solutions provided by the method.

An interesting contrast based region growing approach was introduced in [18]. This method was based on the assumption that the tissue of interest would appear as a bright or dark object relative to the surrounding tissue. This assumption is not valid for our application as there is a tendency to include a part of a blood vessel with the segmented nodule. Therefore, the method fails to address the second feature described above. To solve the reproducibility problem (feature 1), this algorithm can be improved by finding the optimum seed point (the seed with highest intensity). However, since there is no geometrical constraint, the search for the new seed might get diverted into a blood vessel or some lung wall (towards higher intensity points). Further, the method does not make use of any contextual information (feature 5).

Another interesting region growing approach, based on the fuzzy connectivity technique, is introduced in [19]. This technique is based on the path of strongest affinity between each point and the seed point. The concept of a fuzzy connectivity map originated from the work done by Udupa [19]. The fuzzy connectivity algorithm has been used in various medical applications [20]-[23]. This is over-reliant on the setting of detection parameters by the operator, and there are no ideal parameter values which are applicable to all nodule types. In short, the original approach fails to specifically address features 1-6.

An extension of the fuzzy connectivity was introduced in [24] where the description of the relative fuzzy connectivity among different objects was provided. In this approach, the fuzzy connectivity threshold value is not needed (feature 4 solved) since multiple objects would be competing amongst themselves to win the membership of the voxels. However with this approach, both the processing time and user interactivity (feature 6) would be increased as multiple objects would be performing the fuzzy connectivity computations from multiple seed points that need to be provided by the user (feature 3 ). These algorithms are intensity and contrast based region segmentations making no use of contextual information (feature 5). Apart from feature 4, all other issues above still remain unsolved for nodule segmentation.

The method described in this paper exploits the inherent advantages of the two above mentioned region growing techniques, namely fuzzy connectivity [19] and contrast based region growing [18], to create a robust nodule segmentation algorithm. It seeks to address all the above mentioned features. This pulmonary nodule segmentation is aimed at isolated nodules with low and high contrast, nodules with vasculature attachment, and nodules very close to the lung wall or diaphragm.

\section{BRIEF DESCRIPTION OF THE METHOD}

The method performs a contrast based region growing, from a seed point within a fuzzy connectivity map. It outputs the region which has the highest boundary contrast as the extent of the nodule. It also provides alternative segmentation solutions if the first mathematically optimum segmentation result is not to the user's satisfaction. The region growing is confined within a mask that is adaptively obtained. This mask is used to estimate the necessary input parameters of the fuzzy affinity as well as to delineate or separate blood vessels from nodules. The mask is used to seek an optimum seed thus making the algorithm reproducible for any chosen seed location. 


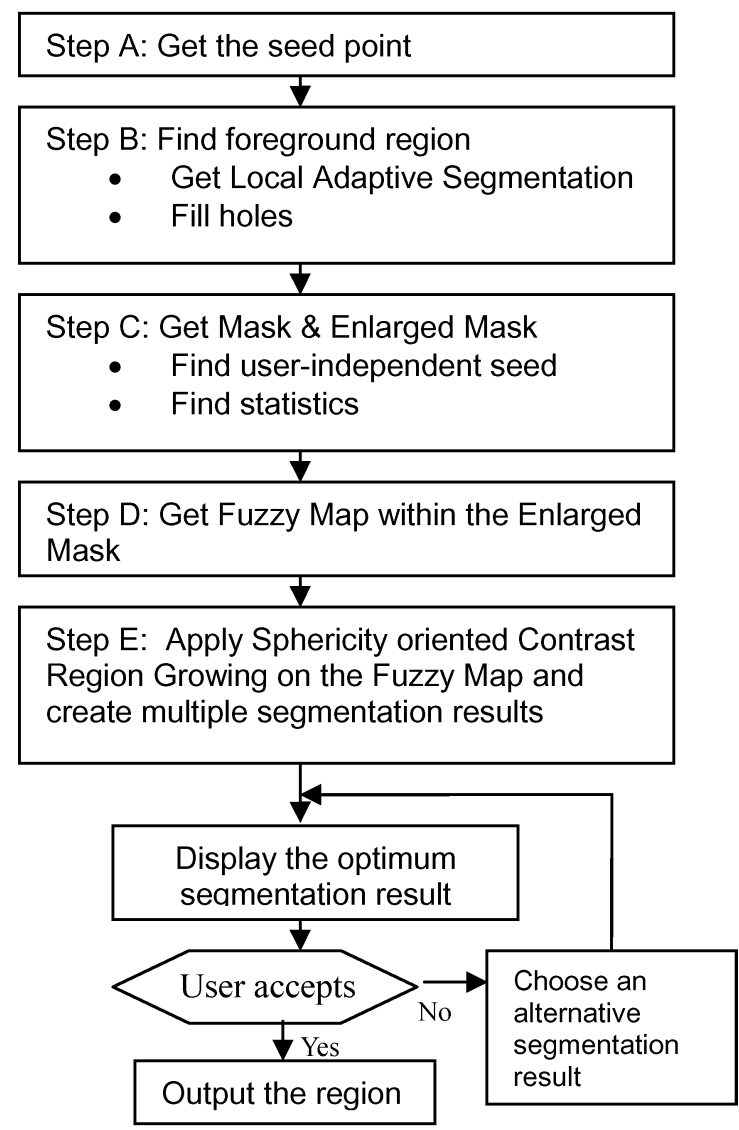

Fig. 1. Flowchart of the algorithm.

Fig. 1 shows the diagram of the algorithm. The different stages of the algorithm are presented as follows.

\section{A. Obtaining a Seed}

A seed, within a nodule, could be selected by the user or it could be obtained through a CAD system.

\section{B. Finding a Foreground Region}

The foreground region is derived through local adaptive segmentation and hole filling procedure as described below.

1) Local Adaptive Segmentation: Local adaptive segmentation is based on local contrast. One of the major advantages is that, in most cases local adaptive segmentation can separate blood vessels or other adjacent tissues from nodules despite very low intensity contrast either between the two objects or their interface, notably without excluding spiculations of the nodule surface. In this case, the local adaptive segmentation can establish a boundary (of the background) between foreground objects of similar intensity. Note that there are several stages proposed in algorithm to separate blood vessel from nodule. Therefore, if the local adaptive segmentation fails to perform properly on a certain nodule, the other components of the algorithm (Sections II-D and II-E) will attempt to identify the proper extent of the nodule.

Another advantage of local adaptive segmentation is that nodules with low contrast can be identified as foreground even when a high intensity object, such as a blood vessel, is just a few pixels distant from the nodule.
Local adaptive segmentation based on local contrast, can lead to spurious detection of foreground objects in background areas of low, but variable intensity. Such variations in background intensity are often due to scanning artifacts such as partial volume effect, motion artifact, or objects that are not likely to be nodules. Hence, the detection of these spurious foreground objects is advantageous because they are then excluded from the mask and do not affect any subsequent processing steps (for instance when adaptively estimating parameters of subsequent steps).

2) Filling Background Holes in the Foreground: Background holes are typically a result of the local adaptive segmentation within objects of high, but variable intensity and do not represent real low-intensity holes. These background holes are filled in and thus converted back into the foreground.

\section{Creating a Mask}

A mask is created by first excluding unconnected foreground objects and then enlarging or inflating the foreground region. The enlargement of the foreground object is made twice its original volume to include a pure background. The mask will be employed to obtain the necessary parameters for the subsequent steps involving two region growing algorithms, and it will also be used as a confined volume for the growing region. The mask will also be utilized in obtaining an optimum seed point. This makes the algorithm reproducible (feature 1).

\section{Constructing a Fuzzy Connectivity Map}

A fuzzy connectivity map is created by applying the fuzzy connectivity region growing technique [19]. The fuzzy connectivity strength is adaptively determined by region growing from the seed point all the way up to the full extent of the mask, created in Section II-C. This implies that predefined fuzzy threshold used in [19] as a halting criteria, is not required (feature 4). The required parameters associated with this step are calculated using the modified expectation maximization (MEM) within the mask. The latter uses a statistical model for the foreground and background material in order to estimate the parameters of the models. Therefore, this technique is adaptive to the contrast of each individual nodule and its immediate local background as defined by the mask. The next step will determine the optimum segmentation solution and provide multiple alternative segmentation solutions based entirely on this fuzzy connectivity map.

\section{E. Sphericity Oriented Region Growing}

This region growing algorithm is applied on the fuzzy connectivity map within the mask. The extent of the nodule is established as the region boundary of the maximum boundary contrast during region growing within the mask (feature 4). This boundary represents the point at which the region is most disconnected from the background, and is therefore most likely to represent the boundary of the nodule (feature 2). Where there is more than one local boundary connectivity contrast maximum, the algorithm suggests the first local maximum and it also provides a facility for the user to choose other solutions (feature 6).

The region growing is weighted according to the strength of connectivity in the connectivity map of each point neighboring the current region. This is so that neighboring points with higher 


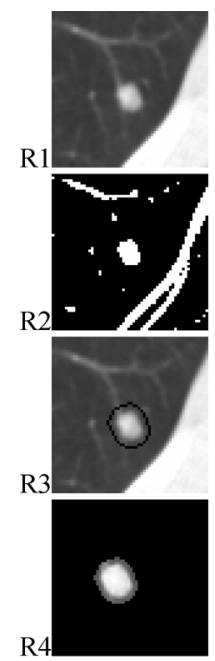

C1

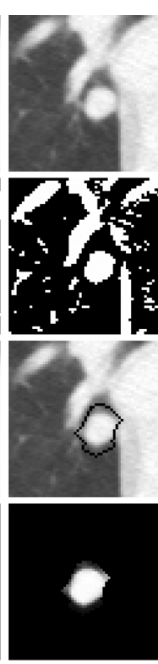

$\mathrm{C} 2$

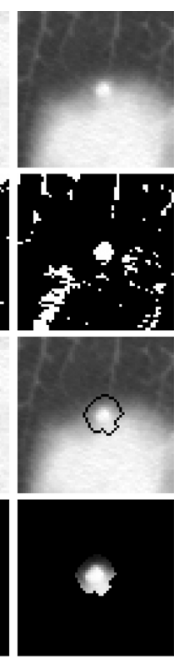

C3

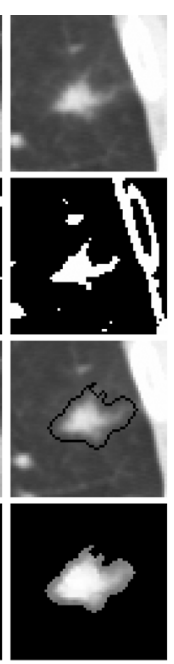

$\mathrm{C} 4$

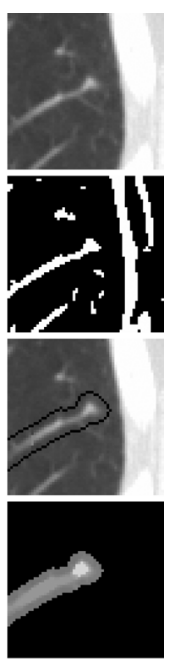

C5
Fig. 2. Different types of pulmonary nodules. C1: Nodule with vasculature attachment. C2: Nodule surrounded by vasculature and lung wall. C3: Small nodule very close to the diaphragm. C4: Irregular shaped and low density contrast nodule. C5: Small low contrast nodule with vasculature attachment. R1: Original image. R2) Local adaptive segmentation. R3: An enlarged mask. R4: Fuzzy map on enlarged mask.

connectivity are added to the region before neighboring points with lower connectivity.

The region growing is also weighted inversely to the distance of each point neighboring the current region from its center. This is so that points closer to the center are added to the region before points further away from the center (hence sphericity oriented region growing). It should be noted that the sphericity criteria is not imposed on region growing as an optimum segmentation result. Instead, it is used to allow for the inclusion of voxels into the dynamically growing regions in a spherical fashion (feature 5).

\section{Detailed Description of Nodule Segmentation}

This section provides a more detailed description of the method used in the proposed algorithm. Fig. 2 illustrates some examples of different pulmonary lung nodules. The intermediate results of the major steps of the proposed algorithm are given in rows $\mathrm{R} 1-\mathrm{R} 4$.

\section{A. Obtaining a Seed Point}

The user of the software inspects the image to identify an area containing a nodule and selects a seed point which appears to be within the nodule. The seed point may be selected automatically as an output of the CAD software.

The user may instead identify a point close to a nodule, but not within it. As detailed below, the method derives an optimum seed point which is insensitive to the precise seed point location chosen by the user even in some cases where the initial seed point is outside but close to a nodule (this is more applicable if the seed is selected by CAD).

\section{B. Finding Foreground Region}

First, a rough estimate of the extent of the region is obtained by means of a local threshold-based segmentation process. A local 3-D volume of about $3 \mathrm{~cm}$ in each direction (for example,
$61 \times 61 \times 21$ pixels at $0.5 \mathrm{~mm} /$ pixel in the $x$ and $y$ directions, $1.5 \mathrm{~mm}$ separation in the $z$ direction), centred on the seed point, is cropped from the whole 3-D image. A local adaptive segmentation algorithm is then performed using a local 3-D mask to segment the image into a plurality of connected objects.

In relation to the implementation, the experiment showed that choosing different mask sizes ranging from $9 \times 9 \times 3$ to $13 \times$ $13 \times 3$ pixels would have a slight effect on the result. However, this would mostly be rectified after the hole filling procedure. A window size of $11 \times 11 \times 3$ was then chosen empirically.

More details in relation to generating the foreground region are provided in the following steps.

1) Local Adaptive Segmentation: The specific algorithm proceeds as follows.

For each pixel within the local volume:

Step 1) define the local mask for that pixel;

Step 2) set the initial threshold intensity as the average intensity within the mask;

Step 3) calculate a histogram of intensity within the mask area;

Step 4) calculate the mean $M 1$ of the part of the histogram below the threshold intensity;

Step 5) calculate the mean $M 2$ of the part of the histogram above the threshold intensity;

Step 6) update the threshold to the mean of $M 1$ and $M 2$;

Step 7) if the threshold is not converged to the mean of M1 and M2 go to Step 4;

Step 8) shift the threshold by a constant intensity value, which is predetermined according to the application;

Step 9) if the intensity of the current pixel is higher than the shifted threshold, define this pixel as foreground; otherwise, define it as background.

The results of the local adaptive segmentation on five different nodules are shown in Fig. 2(R2). As can be seen, the last nodule [Fig. 2(C5)] is not separated from the attached structure. This is a low contrast nodule that is attached to the blood vessel having similar intensity. Although the local adaptive segmentation has failed for this particular nodule, it is detached in the fuzzy map image [Fig. 2(R4,C5)]. As observed, there is a more dense area (a brighter blob) within the center of the nodule as compared to the blood vessel. This is an example that shows the importance of having different stages of the proposed algorithm. The fuzzy map will be discussed in more detail later in Section III-D.

Fig. 3 illustrates four different types of pulmonary nodules with the results of local adaptive segmentation. As demonstrated, the local adaptive segmentation has successfully separated the nodules from the surrounding complex structures in all cases. Note that in Fig. 3(b)-(d), the local adaptive segmentation method has separated the corresponding nodules due to the existence of relatively lower contrast between the nodules and adjacent structures.

2) Filling Holes: The foreground objects acquired by local adaptive segmentation may include "holes" i.e., background pixels surrounded by foreground pixels. This arises because 


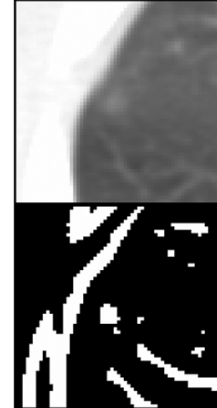

(a)

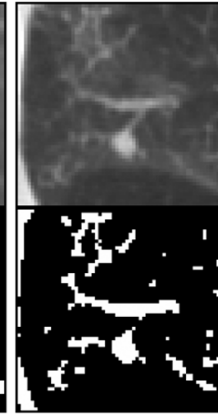

(b)

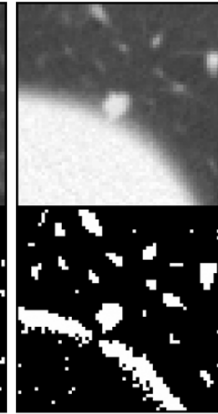

(c)

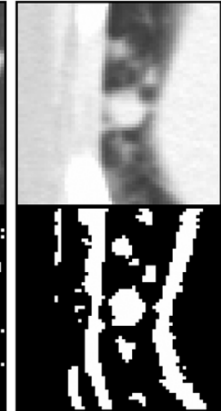

(d)
Fig. 3. Some examples of the local adaptive segmentation which separates the nodule from surrounding complex structures: (a) low contrast nodule; (b) nodule attached to blood vessel; (c) nodule very close to the diaphragm; and d) nodule in-between the lung wall and diaphragm. Note the existence of relatively lower contrast at the interface of the nodules in (b)-(d) and the adjacent tissues. This allows the local adaptive segmentation to separate these nodules from other objects.

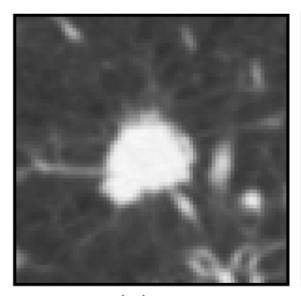

(a)

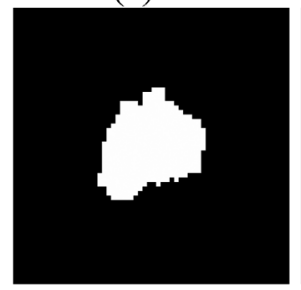

(d)

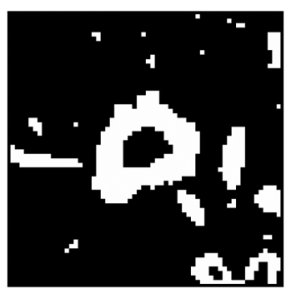

(b)

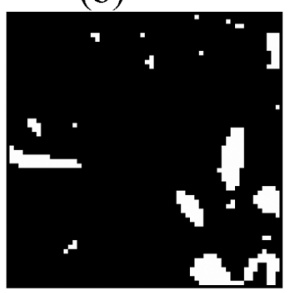

(e)

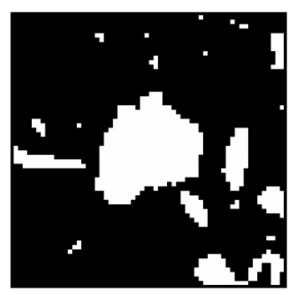

(c)

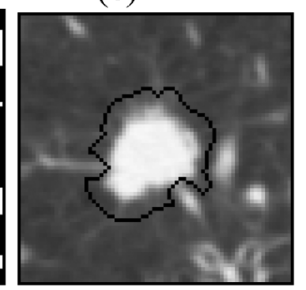

(f)
Fig. 4. Mask region, (a) original image, (b) local adaptive segmentation, (c) holes filled, (d) the chosen region containing seed, (e) unwanted structures, (f) enlarged region of (d) excluding (e).

of the sensitivity of the local adaptive segmentation to small local differences in contrast. Since the foreground objects may represent nodules, they are expected to be solid.

Prior to constructing the 3-D foreground object (Step 3), a 2-D hole-filling algorithm [25] is used to fill in such holes, thus converting them to foreground pixels. A 2-D polygon is fitted to an object containing a hole within a slice, and the pixels within the polygon are all set as foreground. The result of this is shown in Fig. 4(c). The nodule appears as a hollowed object in Fig. 4(b) following the local adaptive segmentation algorithm applied on Fig. 4(a) and the whole nodule is recovered by performing the hole filling procedure.

3) Recovering the Object Containing Seed: The slice which contains the seed point will normally include many segmented regions. The 2-D region which contains the seed point is determined by using a 2-D binary region-growing/labeling scheme [25], as shown in Fig. 4(d).

Once the labelled region has been determined in one slice, a 2-D foreground from an adjacent slice will be connected to the 2-D foreground of the current slice if and only if there is overlap between the displaced core of the current foreground and the foreground of the new adjacent slice. The inclusion of new 2-D foreground regions is applied consecutively through the slices. This leads to the formation of a 3-D foreground region that is defined as $F$. Note that this method of 3-D connectivity is different from the standard 3-D binary labelling [25] where the connectivity is granted by at least a single voxel overlap (or adjacency). The core of an object is obtained in the same manner as for finding the user-independent seed point (described in Section III-C-2).

\section{Finding the Mask and Optimum Seed}

The foreground region $F$ is expanded to serve as a mask and the defining background region for further processing. The mask is also used to obtain an optimum seed. These are described below.

1) Calculating the Mask by Augmenting the Initial Region: The foreground region $F$ is expanded using a 2-D distance transform method to obtain a mask $M$ containing both the foreground and background. This is important, since a lack of background will result in a nonoptimal estimation of parameters used in subsequent region-growing algorithms. The proportion of foreground and background may be approximately equal.

A known 2-D distance transform method is then applied to the inner part of the foreground region, $F$ in each slice, and a maximum distance $S$ to the boundary is obtained, indicating the size of the foreground region. The 2-D distance transform method is later applied to the outer side of the region until the expanded region is twice the area of the foreground region $\mathrm{F}$.

The 3-D distance transform is not recommended because the local adaptive segmentation might have already included low contrast regions at the outer side of the nodules in $Z$ direction (see Figs. 10 and 11). Therefore, any expansion of the object in $Z$ direction may have a wrong impact on the estimation of required parameters in subsequent region growing steps. Particularly, in some cases, it is observed that the expansion in $Z$ direction might include a diaphragm for those nodules being adjacent to it.

Next, the foreground objects segmented by the local adaptive segmentation process that are not labeled as part of the foreground region $F$ are removed from expanded region. The expanded region including the foreground region $F$, but with the unlabelled foreground objects removed, is identified as a mask $M$. A background region $B$ is defined by subtracting the foreground region $F$ from the mask $M$. The mask $M$, the foreground region $F$ and the background region $B$ are used for subsequent calculations. The mask $M$ for the image is shown in Fig. 4(f). The mask is generated by enlarging the foreground region [Fig. 4(d)] and subtracting it from the unwanted objects [Fig. 4(e)]. More examples are provided in Fig. 2(R3).

2) User-Independent Seed Point: As described so far, the definition of the mask $M$ depends on the selection of the seed point by the user. However, in order to get reproducible detection results, a user-independent seed point must be obtained. This is done by defining an iterative process that inspects optimum seed points in the region and which is obtained irrespectively of the intensity distribution within the region. 
At the start of the process, a seed point $S_{o}$ is chosen to be the original seed provided by the user. At each iteration, a mask $M$ will be extracted based on $S_{o}$. A central core or "yolk" of the mask $M$ is found using a distance transform method to erode $k$ layers of the mask $M$. The pixel with highest intensity of the said distance that transforms within the yolk is identified as the following optimum seed point $S_{o}$. The process is repeated until $S_{o}$ converges to the same value.

The process can be summarized in pseudocode as follows:

Siter $=$ Suser

DO

\{

$$
\begin{aligned}
& S_{o}=\text { Siter } \\
& M=\text { Find } \operatorname{Mask}\left(S_{o}\right) \\
& \text { Yolk }=\text { FindYolk }(M) \\
& \text { Siter }=\text { GetMaxIntensityInDistanceMap(Yolk) }
\end{aligned}
$$$$
\text { \} while(So! = Siter }) \text {. }
$$

The optimum seed point $S_{o}$ is taken as the highest intensity point in the 3-D distance map, which is equivalent to the point where the longest line in the $Z$ direction can be traced within the mask. For two similar geometrical regions but with different intensity landscapes, the optimum seed will be the same.

All subsequent steps are performed using the optimum mask $M_{o}$ (the last mask obtained in the process above) and a seed point that could either be the optimum seed $S_{o}$ previously found.

\section{Construct Fuzzy Connected Map}

A fuzzy object extraction technique is used to define a 3-D map of fuzzy connectivity of each pixel within the mask $M_{0}$ with respect to the optimum seed point $S_{O}$. A fuzzy affinity function between adjacent pixels is recognized and the fuzzy connectivity between each pixel and the optimum seed point $S_{O}$ is derived by finding the affinity along a path between the pixel and the optimum seed point $S_{0}$ [19]

The fuzzy connectivity between two points (not necessarily adjacent) is obtained by considering the path with the strongest affinity between two points. The path with the strongest affinity is then chosen as the best path and the strength of each path is equal to that of the weakest affinity of adjacent points along the path.

The affinity between two voxels is a measure of the probability that they belong to the same object. This probability is a function of closeness (i.e., Euclidian distance) and the similarity of the image features (i.e., intensity) between those voxels.

The general model for the fuzzy affinity between two voxels $c$ and $d$ is given as

$$
\begin{aligned}
\mu_{k}(c, d)=\mu_{a}(c, d)\left[\omega_{i} h_{i}(f(c)\right. & f(d)) \\
& \left.+\omega_{g}\left(1.0-h_{g k}(f(c), f(d))\right)\right]
\end{aligned}
$$

where the subscripts $i$ and $g$ represent the calculations related to intensity and gradient values, respectively. $\omega_{i}$ and $\omega_{g}$ are free parameter weight values whose sum is $1 . g k$ is either $g x, g y$, or $g z$, depending on the direction between voxels $c$ and $d . \mu_{\mathrm{a}}$ is an adjacency function based on distance between two voxels which, for $n$-dimensional coordinate voxels, is given by

$$
\mu_{a}(c, d)= \begin{cases}1, & \text { if } \sqrt{\sum_{i=1}^{n}\left(c_{i}-d_{i}\right)^{2}} \leq 1 \\ 0, & \text { otherwise. }\end{cases}
$$

The Gaussian probability function can be used for the affinity

$$
\begin{gathered}
h_{i}(f(c), f(d))=e^{\frac{-(1 / 2)\left[(1 / 2)(f(c)+f(d))-m_{i}\right]^{2}}{\left(S_{i}\right)^{2}}} \\
h_{g k}(f(c), f(d))=e^{\frac{-(1 / 2)\left[(|f(c)-f(d)| / d k)-m_{g k}\right]^{2}}{\left(S_{g k}\right)^{2}}}
\end{gathered}
$$

where $m_{i}$ and $s_{i}$ are the Gaussian parameters for the intensity of the region of interest. $m_{g k}$ and $s_{g k}$ are the Gaussian parameters for the gradient with $k$ being $x, y$, or $z$ depending on the direction between voxels $c$ and $d$. These parameters can be predefined or can be obtained from an initial region as described below. For the current application, the seed point intensity is used for $m i$ and other parameters are estimated within the mask as described in the next section.

1) Estimating the Fuzzy Affinity Parameters: The mean $\mu$ and standard deviation $\sigma$ of the intensity and gradient are calculated over all points within the optimum mask $M_{0}$.

All gradient parameters are computed in three directions, $x, y$, and $z$ separately. For each direction, the corresponding standard deviation is calculated based on the difference between maximum and minimum gradient. $m_{g x}, m_{g y}$, and $m_{g z}$ are taken as the means of the gradients in $x^{-}, y^{-}$, and $z$-direction, respectively, whereas parameters $S_{g x}, S_{g y}$, and $S_{g z}$ are the standard deviation of the gradients in their respective direction.

Adversely, the calculation of the intensity parameters requires more attention as the standard deviation $\left(S_{i}\right)$ appearing in the affinity expression plays a major role in the formation of the fuzzy map and hence the determination of the boundary of the nodule. An accurate estimation can be obtained by assuming that every single pixel within a region belongs to either one or a combination of the following three classes: foreground, partial volume, or background. This schematically can be seen in Fig. 5.

In Fig. 5, the whole range of intensities within a region of interest has been modelled as belonging to the three classes already mentioned. Ranges of image intensities corresponding to each of these classes can be modelled as Gaussian distributions.

This modelling has been carried out through a modified expectation maximization (MEM) algorithm [26]. The following explains the rationale behind MEM for a general case. A special case includes three classes or tissue types: foreground, partial volume, and background.

MEM works on an intensity image $Y=\left\{y_{i}, i=1,2, \ldots, I\right\}$ of region mask with $I$ voxels of intensity $y_{i}$ and $K$ different classes, $\hat{L}=\{1,2, \ldots, K\}$.

A mixed statistical model that takes into consideration spatial properties is employed for the distribution of voxel intensity $p\left(y_{i} \mid \vartheta\right)$ as follows:

$$
p\left(y_{i} \mid \vartheta\right)=\sum_{l \in \hat{L}} a_{l}(i) \cdot p_{l}\left(y_{i} \mid \varphi_{l}\right), \quad i=1,2, \ldots, I
$$




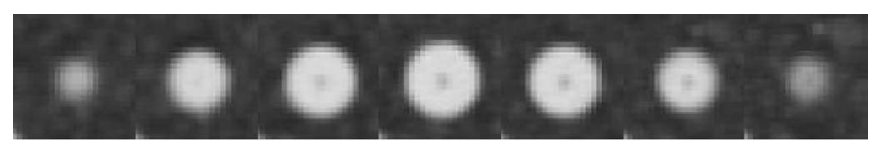

(a)

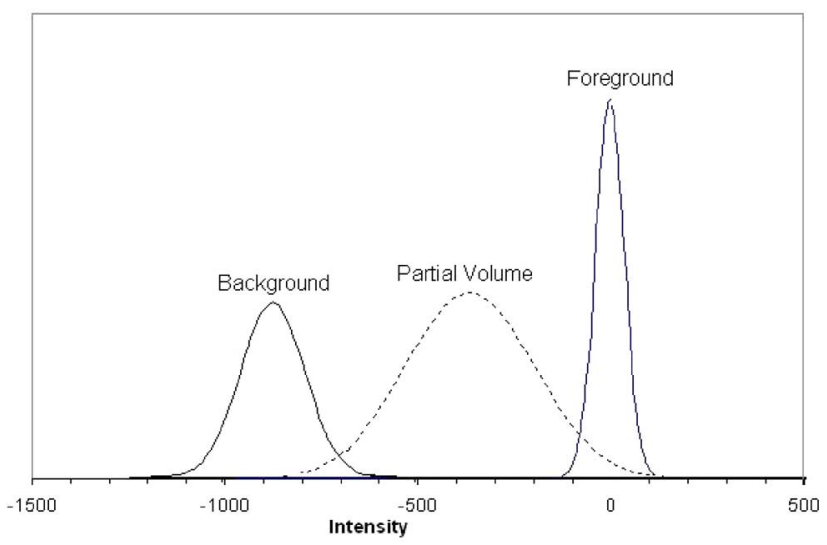

(b)

Fig. 5. Whole range of intensities within a region are modelled through three classes: foreground (F), partial volume (PV), and background (B). Every single pixel within a region will contribute to each of these classes. (a) Cross section images of a nodule from a lung phantom, (b) the modelling of the region in (a) into three classes.

where, for each $i$

$$
p_{l}\left(y_{i} \mid \varphi_{l}\right)=G\left(\mu_{l}, \sigma_{l}\right)=\frac{1}{\sqrt{2 \pi} \sigma_{l}} \exp \left(-\frac{\left(y_{i}-\mu_{l}\right)^{2}}{2 \sigma_{l}^{2}}\right)
$$

which is a Gaussian distribution with parameters $\varphi_{l}=\left(\mu_{l}, \sigma_{l}\right)$ and $\vartheta=\left(a_{1}(i), a_{2}(i), \ldots, a_{K}(i), \varphi_{1}, \varphi_{2}, \ldots, \varphi_{K}\right) . a_{l}(i)$ is a spatial prior probability with spatial constraints imposed by a Markov random field (MRF) and Gibbs random field (MRFGRF) [27].

An MRF $F=\left\{F_{1}, F_{2}, \ldots, F_{k}\right\}$ defined on the set $S$ is a lattice indexing the voxels in the given image $Y$, in which each random variable $F_{i}$ takes a value $l_{i} \in \hat{L}$. The probability density of the MRF $F$ can be given by the Gibbs distribution

$$
p(F)=Z^{-1} \exp [-U(F)]
$$

where $U(F)=\sum_{c \in C} v_{c}(F)$ is the energy function. The energy function is a sum of clique potentials $v_{c}(F)$ over all possible cliques in the enlarged region mask, and $Z$ is a normalization term. According to the Hammersley-Clifford theorem, the conditional probability can be derived using MRF-GRF equivalence as follows:

$$
p\left(l_{i} \mid l_{N(i)}\right)=\frac{\exp \left[-\sum v_{c}\left(l_{i}\right)\right]}{\sum_{l_{i} \in \hat{L}} \exp \left[-\sum v_{c}\left(l_{i}\right)\right]}
$$

where $N(i)$ is the neighbourhood of voxel $i$.

Assuming that the spatial prior distribution $a_{l}(i)$ in (1) is given by the MRF conditional probability $p\left(l_{i} \mid l_{N(i)}\right)$ in (2), the posterior probability $p\left(\varphi_{l} \mid y_{i}\right)$, according to the Bayesian probability theory, can be obtained as

$$
p\left(\varphi_{l} \mid y_{i}\right)=\frac{p_{l}\left(y_{i} \mid \varphi_{l}\right) \cdot p\left(l_{i} \mid l_{N(i)}\right)}{p\left(y_{i} \mid \vartheta\right)} .
$$

Here, the potential function in (2) is defined as

$$
v_{c}\left(l_{i}\right)=\beta \cdot p\left(\varphi_{l} \mid y_{j}\right), \quad j \in c
$$

where $\beta$ is a positive constant which controls the size of clustering. The posterior probability $p\left(\varphi_{l} \mid y_{i}\right)$ represents the probability that the given voxel $i$ belongs to one class $l_{i} \in \hat{L}$. Equation (3) can be used to estimate the highest probability of the reconstructed label image $L$ based on the observed intensity value and the image model as defined in (1) and (2)

The model parameters $\vartheta$ can be obtained to solve (3).

To adapt the model defined in (1) and (2) so that the spatial information is considered by using MRF-GRF model, a modified version of the two-step EM algorithm (i.e., an MEM algorithm) may be used to estimate the parameters of the model and classify voxels of each group simultaneously. For example, for a given $\varphi_{l}^{m}=\left(\mu_{l}^{m}, \sigma_{l}^{m}\right)$, the unique solution $\varphi_{l}^{m+1}=\left(\mu_{l}^{m+1}, \sigma_{l}^{m+1}\right)$ can be derived as

$$
\begin{aligned}
\mu_{l}^{m+1} & =\frac{\left\{\sum_{i=1}^{I} y_{i} \frac{a_{l}^{m}(i) p\left(y_{i} \mid \varphi_{l}^{m}\right)}{p\left(y_{i} \mid \vartheta^{m}\right)}\right\}}{\sum_{i=1}^{I} \frac{a_{l}^{m}(i) p\left(y_{i} \mid \varphi_{l}^{m}\right)}{p\left(y_{i} \mid \vartheta^{m}\right)}} \\
\sigma_{l}^{2(m+1)} & =\frac{\left\{\sum_{i=1}^{I}\left(y_{i}-\mu_{l}^{m+1}\right)^{2} \frac{a_{l}^{m}(i) p\left(y_{i} \mid \varphi_{l}^{m}\right)}{p\left(y_{i} \mid \vartheta^{m}\right)}\right\}}{\sum_{i=1}^{I} \frac{a_{l}^{m}(i) p\left(y_{i} \mid \varphi_{l}^{m}\right)}{p\left(y_{i} \mid \vartheta^{m}\right)}}
\end{aligned}
$$

where $a_{l}^{m}$ in each step can be approximately calculated by assuming

$$
a_{l}^{m}(i) \approx p\left(l_{i} \mid l_{N(i)}\right)=\frac{\exp \left[-\sum v_{c}\left(l_{i}\right)\right]}{\sum_{l_{i} \in \hat{L}} \exp \left[-\sum v_{c}\left(l_{i}\right)\right]} .
$$

The initial values for the MEM parameters are extracted from these different regions: 1) the foreground core region: the original mask $F$ without the internal boundary, 2) partial volume region: the internal and external boundaries of original mask $F$, and 3) the background region (a parenchyma region): the enlarged mask excluding the foreground and the partial volume region.

This MEM process converges after sufficient iterations, and may be halted after a predetermined number of iterations and/or once a predetermined convergence criterion is met. Following this, $S_{i}$ is enhanced by the factor of 5, which was found empirically and was realized through experiments and which is highly tolerant to the variations in the overall results of the current algorithm. The new standard deviation is then used in the fuzzy affinity expression.

2) Fuzzy Map: When the fuzzy threshold is set to zero, the algorithm finds the fuzzy connectivity value for each voxel within the mask $M_{o}$ to the seed point $S_{o}$. This image can be considered as an enhanced image whose voxels represent how strongly they are attached to the seed point $S_{o}$. Fig. 6 shows the fuzzy map images of three different nodules. It should be noted that no mask was used to generate the fuzzy maps. In this example, to illustrate the fuzzy connectivity result with the correct parameters, the mask was used to estimate the statistics, as described in the previous section. 


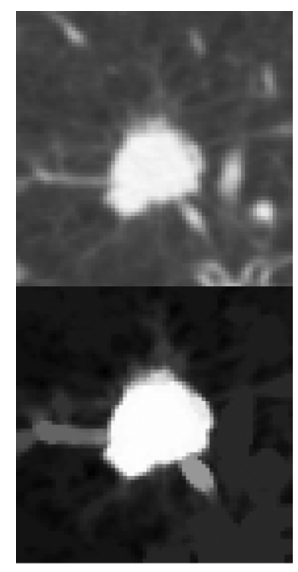

(a)

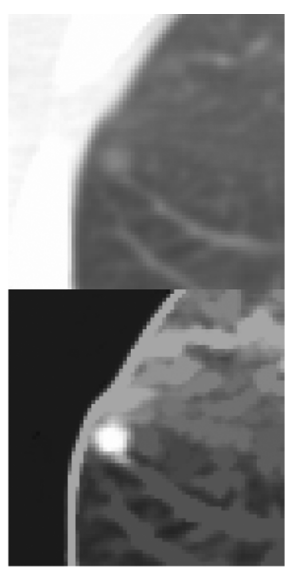

(b)

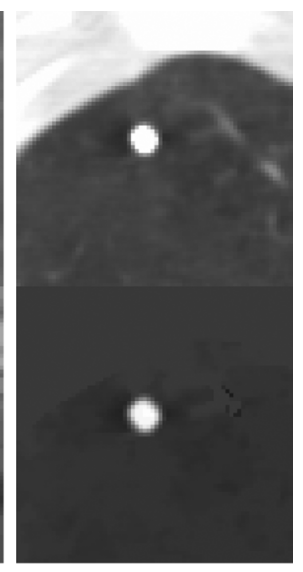

(c)
Fig. 6. Fuzzy map of three different nodules. For each nodule, the fuzzy parameters were estimated using MEM technique within an acquired mask but no mask was imposed to obtain the fuzzy map. (a) Large nodule surrounded by blood vessels. (b) Very low contrast nodule. (c) Calcified (high contrast) nodule.

More examples of the fuzzy map applied on the mask can be found in Fig. 2(R4). The last example [Fig. 2(R4,C5)] illustrates the importance of using fuzzy map since the local adaptive segmentation has failed to detach the nodule from the blood vessel. However, the nodule has been enhanced (and separated from the blood vessel) in the fuzzy map image. This is because, the existence of a relatively lower contrast at the interface of the nodule and blood vessel has an impact on the gradient of the fuzzy affinity measurement. This results in weak fuzzy connectivity strength between the nodule and blood vessel. Therefore, if the local adaptive segmentation fails to perform properly on a certain nodule, the other components of the algorithm (for example, fuzzy map and sphericity oriented contrast based region growing) will attempt to identify the proper extent of the nodule.

\section{E. Sphericity Contrast Based Region Growing on Fuzzy Map}

Instead of setting an arbitrary threshold, the unthresholded fuzzy map can be used as an input to further improve the segmentation. In a preferred embodiment, a parameter-free algorithm using contrast based region growing, is applied to the fuzzy map. In this case, the user only needs to provide the seed point, without threshold adjustment.

To clarify the sphericity contrast-based region growing process, the following terms are introduced.

1) Terminology: The current boundary is the set of pixels adjacent to the current region during the growing process. The internal boundary is defined as the boundary produced by the set of connected outermost pixels of the current region. The current region and the two boundaries dynamically change during the growing process.

The peripheral contrast of the region is defined as the difference between the average grey level of the internal boundary and average grey level of the current boundary

$$
C_{\mathrm{per}}=\bar{f}_{I B}-\bar{f}_{C B}
$$

where $C_{\text {per }}$ is the peripheral contrast of a region, $\bar{f}_{I B}$ is the average fuzzy connectivity of the internal boundary, and $\bar{f}_{C B}$ is the average fuzzy connectivity of the current boundary.
At each iteration of the contrast-based region growing, one pixel is selected from the current boundary and added to the current region. The selection of the priority of pixels in the current boundary is determined on the basis of their intensity and the distance to the center of the current region. The combination of intensity and distance produces a single priority factor $W$.

$$
\begin{aligned}
W & =W_{I} * W_{D} \\
W_{I} & =\frac{1}{1+K 1 *\left(f_{i}-\bar{f}_{F}\right)} \\
W_{D} & =\frac{1}{1+K 2 * D}
\end{aligned}
$$

where $K 1$ and $K 2$ are the weighting factors and are set to $1, D$ is the distance between the candidate pixel and the seed $S_{o}, f_{i}$ is the fuzzy connectivity value of the current voxel, and $\bar{f}_{F}$ is the average fuzzy connectivity of the current region.

When a pixel is added into the current boundary, the internal boundary and the current boundary are updated. The peripheral contrast is then calculated and added to a vector which relates to the current region size. This process continues until the region fills the extended mask $M_{o}$. As region growing expands into the total space within the mask, there might be several maximum peripheral solutions, which represents multiple segmentation results.

The first maximum peripheral contrast value obtained during region growing is selected as indicating the optimum region, with a boundary most likely to correspond to that of the nodule. This optimum boundary is then output as the detected boundary of the nodule. In case the first segmentation result is not to the user's satisfaction, the user can select alternative multiple segmentation results that are available.

2) Sphericity Oriented Contrast Based Region Growing Algorithm: The algorithm for contrast based region growing is as follows.

Step 1) Define $M_{O}$ as a maximum region size.

Step 2) Select the seed point $S_{0}$ and add it to the current region.

Step 3) Use second order connectivity (eight neighbors for 2-D and 26 for 3-D) to find neighbors and first order connectivity (four for 2-D, six for 3-D) to find/update the current boundary. Sort the points in the current boundary in ascending order by using the priority factor given by (6).

Step 4) Find/update the internal boundary.

Step 5) Calculate the peripheral contrast $\left(C_{\text {per }}\right)$ and put the value in the peripheral contrast vector.

Step 6) Find the point with the highest priority $W$ [from (6)] in the current boundary and add to the current region. If the region reaches the predefined maximum size (Mask size), go to Step 7, otherwise go to Step 3.

Step 7) In the peripheral contrast vector, find the first maximum value (local maximum) and the corresponding region size, and output the current region. The boundary of the current region is taken as the extent of the nodule (optimum segmentation result). 


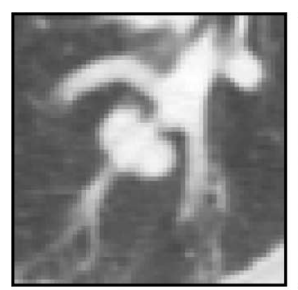

(a)

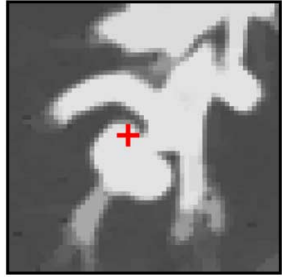

(b)

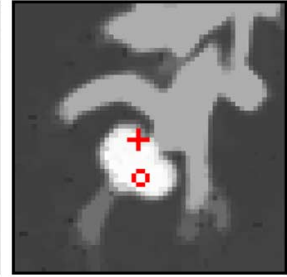

(c)
Fig. 7. Effect of seed position on a nodule attached to blood vessel. (a) Original image. (b) Fuzzy map grown from the initial seed (marked with a cross). (c) Fuzzy map grown from the optimum seed (shown as circled). Contrast between the nodule and the blood vessel is more visible when the optimum seed is used.

Step 8) Record all the local maximums found on the peripheral contrast vector in a descending order to provide multiple segmentation results.

\section{EXPERIMENTAL RESULT}

Obtaining a ground truth (i.e., a "gold standard") for defining the border of nodules in clinical data is challenging. There can be interobserver and intraobserver differences in manually outlined nodules provided by experts. Consequently, validating the acceptability of any nodule segmentation algorithm is subjective. Given the above constraint, the segmentation results of the current algorithm have been visually inspected by a qualified radiologist for any gross missegmentation, such as a failure in separating nodules from vasculature. For a gross misssegmentation, the radiologist selected other alternative segmentation solutions provided by the algorithm (Steps 7 and 8).

It should be noted that given a large number of algorithms published in the literature, it is not feasible to provide a comparative study based on a common database. Since the current algorithm is a region growing based method that utilizes fuzzy connectivity [19] and contrast region growing [18], the first part of this section provides comparative studies with these two methods. This part also shows the importance of using the different stages of the algorithm. The second part provides the experimental results of the algorithm applied on 815 nodules.

\section{A. Comparative Results}

Before presenting the comparative results, the importance of using the optimum seed rather than the initial seed is illustrated in Fig. 7. For this experiment, the mask was used to find the optimum seed (indicated as a small circle), and no mask was employed for the region growing, in order to show the fuzzy connectivity between the seed and all the surrounding structures. In both cases of Fig. 7, the same initial seed point, indicated with a cross, was used. In the first experiment [Fig. 7(b)], then the fuzzy map was generated from the initial seed point; while in the second experiment [Fig. 7(c)], the seed was first moved to an optimum position and the fuzzy map was created. As can be seen, the contrast between the nodule and the blood vessel is more noticeable when the optimum seed is used. This results in more accurate nodule segmentation as well as making it fully reproducible. The reproducibility test will be provided in Section IV-B of the experimental results.
Note that for the following comparative experiment, the seed positions were chosen such that the best results were obtained using methods in [18] and [19]. For the proposed algorithm, the initial seed positions were not important, as optimum seed points were obtained as part of the algorithm. This was described in Section III-C.

Fig. 8 shows a comparison of different techniques on four different nodules. The results of the classical fuzzy connectivity region growing are shown in Fig. 8 (R2)-(R5). These experiments were conducted to illustrate the sensitivity of the fuzzy connectivity region growing, proposed in [19], on the parameters. No masks were used for these cases. Fig. 8(R2) and (R3) displays the effect of the standard deviation in the fuzzy affinity expression (Section III-D) on the segmentation result. In Fig. 8(R2), the standard deviation was set to produce the best result for the first nodule [Fig. 8(R2,C1)]. This parameter was too aggressive for the other three nodules, which resulted in the inclusion of adjacent tissues in segmentation result. A lower standard deviation (the same fuzzy threshold) was then used to allow for a proper segmentation for the second nodule, as shown in Fig. 8(R3,C2). This lead to an under-segmentation of the first nodule, an acceptable segmentation of the third nodule and a failure of the last one. These results prove that an adaptive approach for estimating standard deviation per nodule is required. The proposed algorithm uses MEM method to overcome this problem (Step 1 of Section III-D).

Fig. 8(R4) and (R5) displays the effect of the fuzzy threshold (Step 2 in Section III-D) on the segmentation result. For these experiments, the standard deviations for each nodule were estimated using MEM (as described in Step 1 in Section III-D). Two arbitrary fuzzy threshold values were used to obtain segmentation results as displayed in Fig. 8(R4) and (R5). As can be seen, the lower threshold of Fig. 8(R4) produced proper segmentations for the first two nodules while it failed on the third and the fourth nodules. Conversely, the fuzzy threshold with the higher value, Fig. 8(R5), was sufficient for the second and the third nodules but failed on the fourth and it was also under-segmented for the first nodule. These results clearly indicate that fuzzy connectivity can be sensitive to the fuzzy threshold value. This problem has been solved in the current algorithm by applying the sphericity oriented contrast based region growing on the fuzzy map as explained in Section III-E.

Fig. 8(R6) and (R7) shows a comparative result of the original contrast based region growing [18] against the sphericity oriented contrast based region described in Section III-E. Both methods were applied on the raw images of Fig. 8(R1). The sphericity constraint contrast based region growing [i.e., $k 2=1$ in (8)] performed well on the first nodule as compared to the original contrast based region growing $[k 2=0$ in (8)]. The performance was also slightly better on the second nodule and, for the third and fourth nodules both techniques failed to segment the nodules due to the strong presence of a blood vessel and the nodule being too close to the diaphragm respectively.

In order to further illustrate the effect of the sphericity constraint on contrast based region growing, a schematic smoothed graph of peripheral contrast (see Section III-E) against the region size for the first nodule [Fig. 8(C1)] was generated and this is shown in Fig. 9. The region corresponding to the max- 


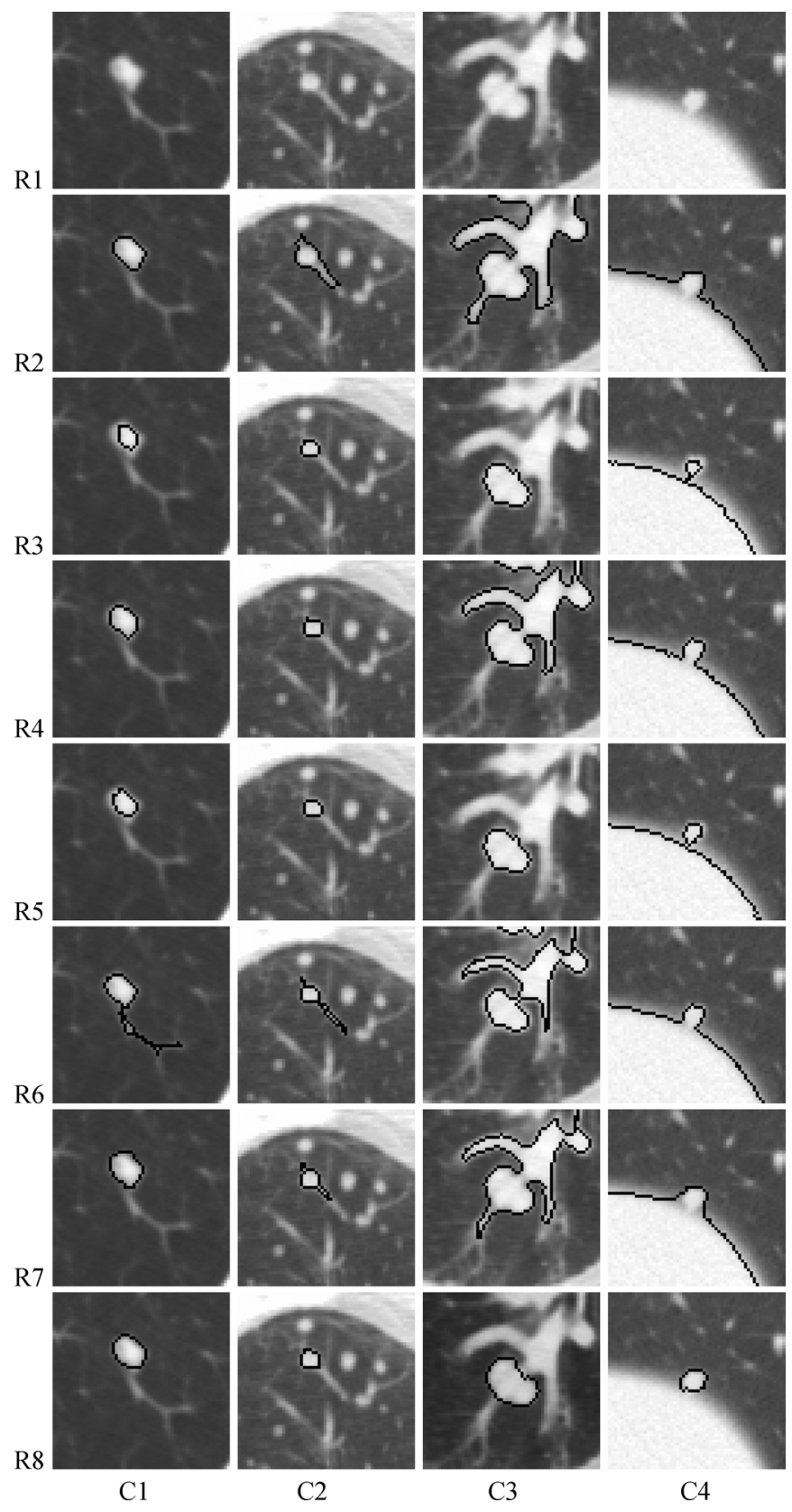

Fig. 8. Results of the four techniques on four different types of pulmonary nodules (columns $\mathrm{C} 1-\mathrm{C} 4$ ). Rows indicate different tests on the given nodules. R1: Original image. R2: Results of the classical fuzzy region growing with no mask. R3: Same as R2 with lower standard deviation and the same fuzzy threshold. R4: Results of the classical fuzzy region growing with no mask using estimated standard deviation from MEM. R5: Same as R4 with higher fuzzy threshold. R6: Results of the contrast based region growing (no sphericity constraint) with no mask. R7: Results of the sphericity oriented contrast based region growing with no mask. R8: Results of the proposed method.

imum peaks on the graph indicates the optimum segmentation result as given (outlined) in Fig. 8(R6,C1) and 8(R7,C1). This is a nodule attached to a blood vessel. Fig. 8(R6,C1) shows the result of region growing corresponding to the maximum peak of the dotted line graph of Fig. 9. This result is obtained when the region growing is weighted according to the strength of connectivity in the intensity map of each point neighboring the current region $\left[k_{1}=1 ; k_{2}=0\right.$ in (6)-(8)], so that those neighboring

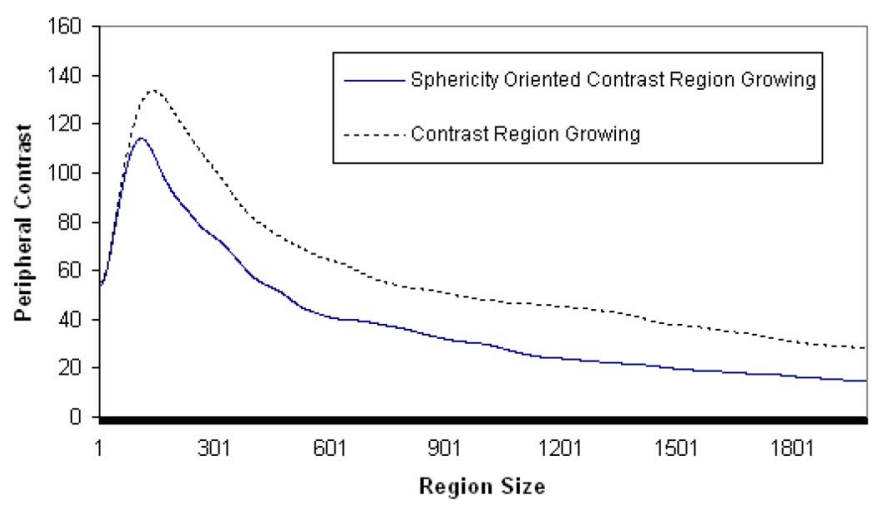

Fig. 9. Peripheral contrast versus number of pixels for nodules of Fig. 8(R6,C1) and $(\mathrm{R} 7, \mathrm{C} 1)$. Region corresponding to the maximum peripheral contrast, the peak, is taken as the nodule segmentation result. Peak has slightly shifted in the sphericity oriented region growing. In this typical example, using the sphericity oriented region growing, the peak of the contrast profile occurs before region growing fills the blood vessel, resulting in successful segmentation of the nodule of Fig. 8(R7,C1).

points with higher connectivity are added to the region before neighboring points with lower connectivity. As a result, the region growing fills a part of the blood vessel before filling in the outer boundary of the nodule. Fig. 8(R7,C1) shows the result of region growing corresponding to the maximum peak in the solid line graph of Fig. 9. This result is obtained when the region growing is also weighted inversely to the distance of each point neighboring the current region from its center $\left(k_{1}=1, k_{2}=1\right)$ so that points closer to the center are added to the region before points further away. As a result, the peak of the solid line graph in Fig. 9 slightly shifts to the left, as compared to the peak of the dotted line, and the desired peak is achieved before region growing fills the blood vessel, as shown in Fig. 8(R7,C1).

Finally, the results of the proposed algorithm on the same nodules are provided in Fig. 8(R8). All the nodules are correctly delineated from the surrounding structures without using any predefined parameters. It should be noted that the same results were obtained with different initial seed points. With the proposed algorithm, the extracted boundaries cover the totality of the nodules more sufficiently as compared to other algorithms ([18] and [19]).

The last nodule [Fig. 8(C4)] is a low contrast structure and it is almost attached to the diaphragm. As seen, the proposed algorithm has successfully extracted the nodule, while the other algorithms have failed to separate the nodule from the diaphragm. In this case, the local adaptive segmentation described in Section III-B has managed to separate the nodule from the lung wall, as was shown in Fig. 3(c).

\section{B. Reproducibility and Acceptability of the Proposed Method}

The previous section described and illustrated the comparative results between the proposed method and the classical fuzzy connectivity [19] and contrast region growing [18]. The aim of this experiment is now focused on evaluating the proposed method using extensive data sets. The following are details of the two databases that were used in this study. For both databases, nodules ranging between 5 and $30 \mathrm{~mm}$ in diameter were chosen. 
Database1: This database was used during the research and development (RD) of the algorithm. It consists of 608 pulmonary nodules out of 343 scans with a slice thickness ranging from 0.5 to $2.0 \mathrm{~mm}$ and the $\mathrm{X}$-ray tube current ranging from 80 to $300 \mathrm{~mA}$. Out of 608 pulmonary nodules, 351 were isolated nodules with no significant connection to vasculature. This included solid or high contrast nodules (having CT numbers much higher than that of surrounding voxels) and low contrast nodules (having CT numbers closer to those of surrounding voxels). 257 of 608 nodules are being either attached to blood vessel or near lung wall or diaphragm.

Database 2: This database was available as an independent testing group. It contained 207 pulmonary nodules out of $80 \mathrm{CT}$ scans obtained from two centers. 50 of those CT scans were acquired in Center 1 using a protocol of $3.0 \mathrm{~mm}$ slice thickness and $200 \mathrm{~mA} \mathrm{X}$-ray tube current. The remaining 30 scans were acquired in Center 2 with a protocol of $2.0 \mathrm{~mm}$ and $250 \mathrm{~mA}$ for slice thickness and $\mathrm{X}$-ray tube current, respectively. The database contained 109 isolated nodules and 98 vascularized nodules or nodules near the lung wall or diaphragm.

1) Reproducibility Evaluation: The aim of this experiment is to examine whether the algorithm produces the same optimum seed point from different initial seed points. This experiment is carried out regardless of the acceptability of the segmented regions which will be the aim of the next sections.

To evaluate this reproducibility experiment efficiently, a simple software interface was designed to allow the user to provide approximate areas that would roughly mark the boundaries of the nodules. It should be noted that the marked areas only represent approximate regions that might not accurately cover the whole portion of the nodules. As such, they were not used to examine the acceptability of the segmented regions of the algorithm. They were only used to automatically determine random seed points to perform the reproducibility test for the given method. As a result, an automated software tool was designed as described below.

For each nodule, 10 random seed points were obtained within the corresponding marked area. The algorithm was applied to each random point to obtain the optimum seed point. If the algorithm produced the same optimum seed location for all the 10 random points (for each nodule), the method was considered to be successful in performing this stage of the algorithm, namely the optimum seed generation.

This automated software tool was applied on both the databases (Database1 and Database2) containing a total of 815 pulmonary nodules. The software tool produced a successful outcome for all 815 cases. This indicates that the proposed algorithm is fully reproducible because it can produce the same optimum seed point from different initial seeds, hence leading to the same segmentation result.

Fig. 10 shows an example of the above experiment. The results from 10 random seed points were overlaid on the same cross section images. The positions of the ten random seed points are displayed in the images. As the figure illustrates, all ten attempts produced exactly the same optimum seed point (shown as a darker point and marked with an arrow). This leads to the same segmentation result as shown in the figure (which also shows the boundary of the segmentation result).

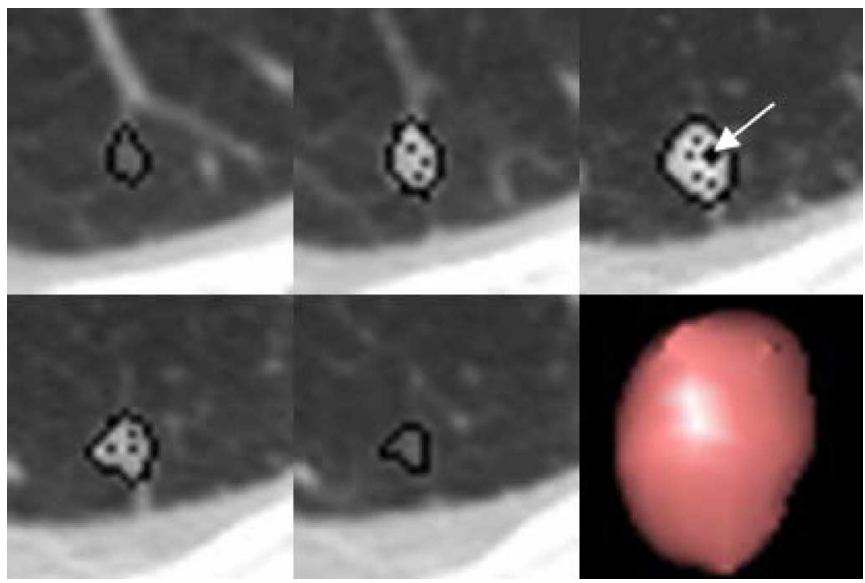

Fig. 10. Positions of the ten random seed points are displayed as black dots inside the nodule, while the optimum seed is shown as a larger dot with an arrow. All the random seed points lead to same optimum point and therefore produced exactly the same segmentation result. Bottom-right image shows the 3-D surface rendering of the extracted nodule.

2) Acceptability Evaluation Using Database1: For this experiment, Database1 was used to evaluate the acceptability of the method. The acceptability of the extracted nodules was examined by a qualified radiologist based on visual inspection for any gross misssegmentation. For a gross misssegmentation, such as a failure in separating nodules from adjacent tissue, the radiologist selected other alternative segmentation solutions provided by the algorithm. The radiologist reported that out of 608 nodules, $85 \%$ were considered accurately segmented. This means that the radiologist was satisfied with the first optimum segmentation result offered by the proposed algorithm. For the remaining $15 \%$, the radiologist chose an alternative segmentation solution. All segmentation results that required an alternative segmentation result were part of the vascularized nodules or nodules very close to the lung wall or diaphragm.

Fig. 11 illustrates two examples of difficult nodules from the above experiment. The radiologist has confirmed that they have been successfully extracted. A volume rendering is provided to display the complexity of the structures that are attached to the nodules.

Fig. 12 shows an example of a typical nodule, very close to a wall, with its multiple segmentation results provided by the proposed algorithm. Fig. 12(a) illustrates the occurrence of multiple peaks on the peripheral contrast curve which represent multiple solution results. Fig. 12(b) shows an alternative segmentation solution (that corresponds to the peak B) generated by the algorithm. This was accepted by the user while the original segmentation solution (at peak A) was rejected.

3) Acceptability Evaluation Using Database2: The segmentation results of the current method on 207 pulmonary nodules from Database2 were visually inspected by a qualified radiologist. The results indicated that out of all 207 nodules, 83\% were categorized as accurately segmented (first best solution provided by the algorithm), and $17 \%$ required an alternative solution provided by the algorithm. All the alternative segmentation results selected by user were from the vascularized nodules or nodules very close to the lung wall or diaphragm. 

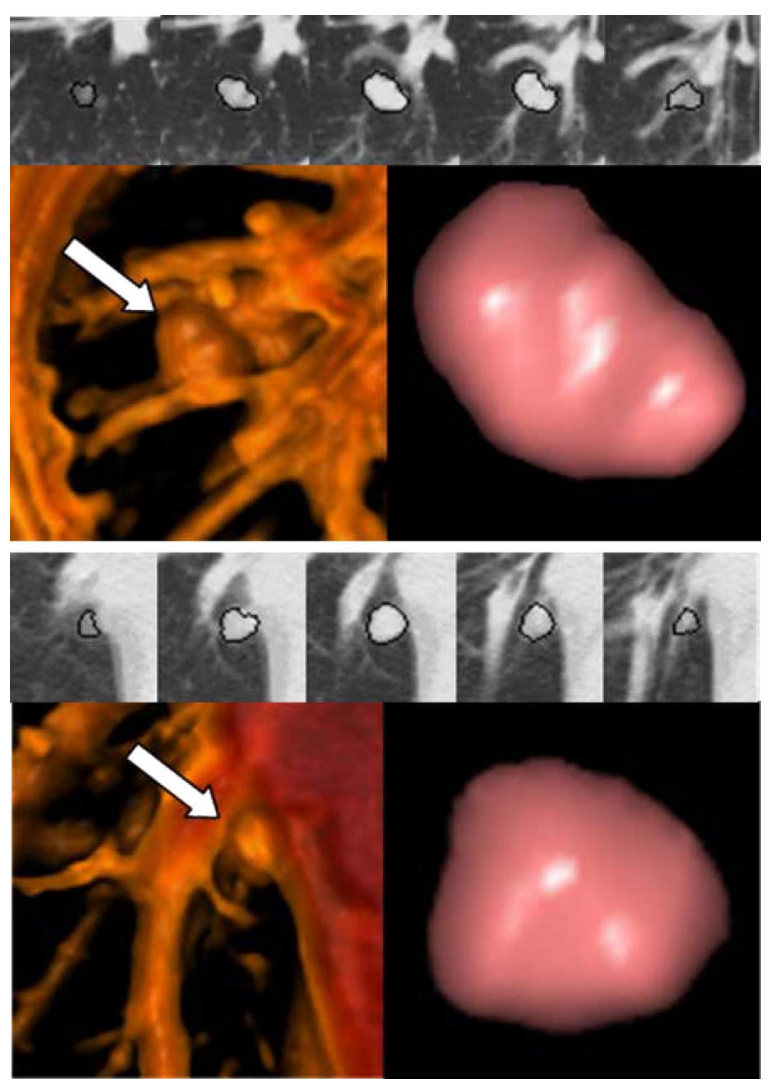

Fig. 11. Three-dimensional region growing of two nodules using the proposed algorithm. For each case, the top image shows the boundary while the bottom image gives the 3-D volumetric (left image) and surface rendering result (right images). White arrow is drawn to highlight the position of the nodules in the 3-D volumetric images.

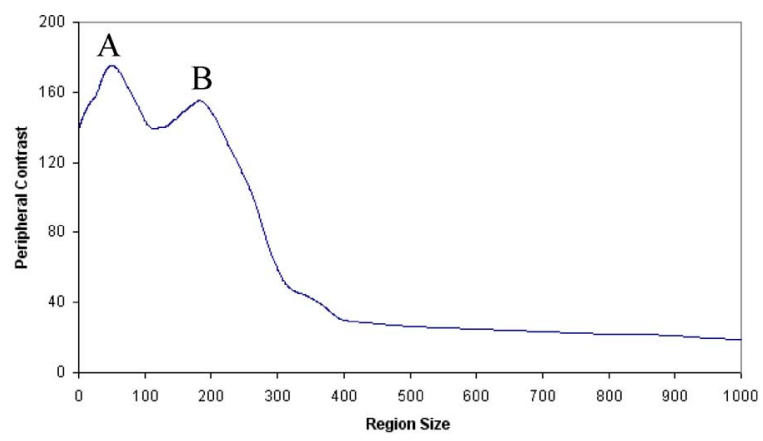

(a)

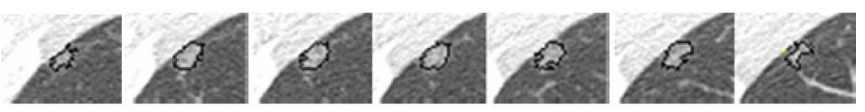

(b)

Fig. 12. Example of a nodule with multiple segmentation solutions. (a) Graph for the peripheral contrast versus number of pixels showing multiple peaks. (b) Segmentation result corresponding to the peak B where the first segmentation result (at peak A) was rejected.

\section{DISCUSSION}

This paper has presented an efficient region growing algorithm that is highly reproducible and is able to segment different types of pulmonary nodules including high and low contrast nodules, nodules with vasculature attachment, and nodules very close to the lung wall or diaphragm.

The algorithm was designed to address the features 1-6 that were presented in Section I. Each of the features is reiterated in this section.

\section{A. Seed Point Sensitivity}

The method has found an optimum seed point prior to performing the region growing for nodule segmentation. This has been done to make the method reproducible for any initial seed point (within nodules) that is chosen by the user. The experimental results section has provided the outcome of the reproducibility test on 815 pulmonary nodules. It has shown that the current method is not sensitive to the initial placement of seed points.

\section{B. Delineation of Nodule Boundary}

The experimental results have shown that the algorithm is successful in that it is able to detect the extent of all isolated nodules including high and low contrast nodules (460 out of 815) and required no adjustment (i.e., selecting alternative segmentation solution).

A combination of the three components (local adaptive segmentation, fuzzy connectivity map, and sphericity oriented region growing) has produced a successful separation of many vascularized nodules, or nodules very close to lung wall or diaphragm.

The first two components are based on the premise that voxels residing at the junction of the nodules (whether it has a regular or irregular shape) and blood vessels (or other adjacent objects), often have lower intensity values as compared to those with the denser structures such as the blood vessel. As a result, in the local adaptive segmentation algorithm, the interface voxels have been forced back to zero (background) which results in separating the blood vessel from the nodule. Further, lower intensity values will have an impact on the gradient of the fuzzy affinity measurement which might result in weak fuzzy connectivity strength between the two objects (nodule and blood vessel). Our observation and experimental results have shown no trace of such low intensity values (interfaces) between the compact area of an irregular shaped nodule and its irregular surface (such as its spiculation). In fact, the intensity values of an irregular nodule either gradually decreases from its very compact area towards its surface detail or have similar intensity values (with no low intensity value interface). However, we do not discard the possibility that there might be an odd occurrence of lower intensity at the interface of such irregular spiculated nodules; in such cases our method might inaccurately remove the surface detail of the nodule.

The third component, the sphericity oriented region growing using contextual information for the nodule segmentation, is discussed in the Section V-E.

The experimental results have indicated that 231 nodules out of 355 vascularized nodules and nodules very close to the lung wall or diaphragm were successfully segmented using the first best solution provided by the algorithm. The remaining 124 out of 355 of nodules have required alternative segmentation solutions provided by the algorithm. 


\section{Few Number of Input Parameters and Use of Adaptive Parameter Setting}

The only input parameter given by the user is a seed point and other parameters have been adaptively estimated per nodule, as described in Section III-D.

\section{Halting Criteria}

The current method does not require any threshold setting as the halting criteria. Instead, it uses a sphericity oriented contrastbased region growing technique (see Section III-E) on the fuzzy map (see Section III-D) within a mask to adaptively identify the extent of a nodule.

\section{E. Morphology}

The sphericity oriented region growing takes into account the spherical shape of a nodule by encouraging points closer to the center to be added to the region before points further away from the center are added. (see Section III-E).

It should be noted that the sphericity criteria has not been imposed on region growing as an optimum segmentation result or used as a halting criteria to get the region growing algorithm to stop at a specific boundary with spherical shape. Instead, it has allowed voxels to be added to the growing region in a spherical fashion. This growing process continues until the region fills the mask. The direction of the growing process within the mask has caused the region to grow first on the very compact part of the nodule, next towards the edge, later towards the surface detail of the nodule (such as spiculations) and finally to any blood vessel (i.e., if other components of the algorithm fail to separate the blood vessels attached to nodules, as explained in Section V-B). This has increased the likelihood of a first local maximum boundary connectivity contrast taking place before the region grows incorrectly to adjacent tissues with high connectivity within the mask. The advantage of using the sphericity oriented contrast region growing technique has been shown in Fig. 8(R7,C1).

As region growing expands into the total space within the mask, there might be several maximum peripheral solutions, which represent multiple segmentation results.

\section{F. Usability}

The method is optimized to reduce the computation time. For example, it takes $5 \mathrm{~s}$ in average to run the method on a $2 \mathrm{GHz}$ Pentium 4 with a 2 GB RAM. Furthermore, the method requires only a single seed point and allows for multiple segmentation results in case the first given segmentation solutions are not to the satisfaction of the user.

\section{CONCLUSION}

A detection of the extent of a lung nodule (nodule segmentation) is vital for volumetric measurement of nodules and hence an accurate diagnosis. For this reason, a new region growing method has been proposed for lung nodule segmentation. This method uses a combination of fuzzy connectivity, distance and intensity information as the growing mechanism and peripheral contrast as the halting criterion. After comprehensive tests with real images, it was found that this region growing method could provide encouraging results for pulmonary nodules in CT lung images. The results also indicate that the method is highly reproducible for various types of nodules from various data protocols.

\section{ACKNOWLEDGMENT}

The authors would like to thank A. Hashemi and F. Dehmeshki for their constant help with the evaluation of the datasets, all radiologists who took part in this study, and Dr. H. Rodrigues for proof reading. This work was carried out at Medicsight Plc.

\section{REFERENCES}

[1] Y. Lee, T. Hara, H. Fujita, S. Itoh, and T. Ishigaki, "Automated detection of pulmonary nodules in helical CT images based on an improved template-matching technique," IEEE Trans. Med. Imag., vol. 20, no. 7, pp. 595-604, Jul. 2001

[2] B. Zhao, G. Gamsu, M. S. Ginsberg, L. Jiang, and H. Schwartz, "Automatic detection of small lung nodules on CT utilizing a local density maximum algorithm," J. Appl. Clin. Med. Phys., vol. 4, no. 3, pp. 248-260, 2003.

[3] K. Suzuki, S. G. Armota, F. Li, S. Sone, and K. Doi, "Massive training artificial neural network (MTANN) for reduction of false positives in computerized detection of lung nodules in low-dose CT," Med. Phys., vol. 30, no. 7, pp. 1602-1617, Jul. 2003.

[4] Z. Y. Ge, B. Sahiner, H. P. Chan, L. M. Hadjiiski, P. N. Cascade, N. Bogot, E. A. Kazerooni, J. Wei, and C. Zou, "Computer-aided detection of lung nodules: False positive reduction using a 3-D gradient field method and 3-D ellipsoid fitting," Med. Phys., vol. 32, pp. 2443-2454, 2005.

[5] M. S. Brown, M. F. McNitt-Gray, J. G. Goldin, R. D. Suh, J. W. Sayre, and D. R. Aberle, "Patient-specific models for lung nodule detection and surveillancein CT images," IEEE Trans. Med. Imag., no. 12, pp. 1242-1250, Dec. 2001

[6] C. I. Fetita, F. Prêteux, C. Beigelman-Aubry, and P. Grenier, "3-D automated lung nodule segmentation in HRCT," in Medical Image Computing and Computer-Assisted Intervention-MICCAI. New York: Springer, pp. 626-634.

[7] J. P. Ko, H. Rusinek, E. L. Jacobs, J. S. Babb, M. Betke, G. McGuinness, and D. P. Naidich, "Small pulmonary nodules: Volume measurement at chest CT-Phantom study," Radiology, vol. 228, pp. 864-870, 2003.

[8] N. Xu, N. Ahuja, and R. Bansal, "Automated lung nodule segmentation using dynamic programming and EM based classification," Proc. SPIE, vol. 4684, pp. 666-676, 2002.

[9] Y. Kawata, N. Niki, H. Ohmatsu, R. Kakinuma, K. Eguchi, M. Kaneko, and N. Moriyama, "Quantitative surface characterization of pulmonary nodules based on thin-section CT images," IEEE Trans. Nucl. Sci., vol. 45, no. 4, pp. 2132-2138, Aug. 1998.

[10] Y. Kawata, N. Noboru, H. Ohmatsu, and N. Moriyama, "A deformable surface model based on boundary and region information for pulmonary nodule segmentation from 3-D thoracic CT images," IEICE Trans. Inf. Syst., vol. E86-D, pp. 1921-1930, 2003.

[11] W. Mullally, M. Betke, J. Wang, and J. P. Ko, "Segmentation of nodules on chest computed tomography for growth assessment," Med. Phys., vol. 31, no. 4, pp. 839-848, Apr. 2004.

[12] L. Fan, J. Qian, B. L. Odry, and H. Shen, "Automatic segmentation of pulmonary nodules by using dynamic 3-D cross-correlation for interactive CAD systems," in Proc. SPIE Med. Imag., 2002, vol. 4684, pp. 1362-1369.

[13] K. Okada, D. Comaniciu, and A. Krishnan, "Robust aniostropic gaussian fitting for columetric characterization of pulmonary nodules in multislice ct," IEEE Trans. Med. Imag., vol. 24, no. 3, pp. 409-423, Mar. 2005

[14] K. Okada, U. Akdemir, and A. Krishnan, "Blob segmentation using joint space-intensity likelihood ratio test: Application to 3-D tumor segmentation," in IEEE Conf. Comput. Vis. Pattern Recognit., Jun. 2005, no. 2, pp. 437-444.

[15] W. J. Kostis, A. P. Reeves, D. F. Yankelevitz, and C. I. Henschke, "Three-dimensional segmentation and growth-rate estimation of small pulmonary nodules in helical CT images," IEEE Trans. Med. Imag., vol. 22 , no. 10 , pp. $1259-1274$, Oct. 2003. 
[16] B. Zhao, D. Yankelevitz, A. P. Reeves, and C. T. Henschke, "Two-dimensional multi-criterion segmentation of pulmonary nodules on helical CT images," Med. Phys., vol. 26, no. 6, pp. 889-895, Jun. 1999.

[17] B. Zhao, A. P. Reeves, D. F. Yankelevitz, and C. T. Henshcke, "Threedimensional multicriterion automatic segmentation of pulmonary nodules of helical computed tomography images," Opt. Eng., vol. 38, pp. $1340-1347,1999$

[18] S. A. Hijjatoleslami and J. Kitter, "Region growing: A new approach," IEEE Trans. Image Process., vol. 7, no. 7, pp. 1079-1084, Jul. 1998.

[19] J. K. Udupa and S. Samarasekera, "Fuzzy connectedness and object delineation: Theory, algorithm, and validation," Graph. Models Image Process., vol. 58, no. 3, pp. 246-261, 1996.

[20] J. K. Udupa, L. Wei, S. Samarasekera, Y. Miki, M. A. Buchem, and R. I. Grossman, "Multiple sclerosis lesion quantification using fuzzyconnectedness principles," IEEE Trans Med. Imag., vol. 16, no. 5, pp. 598-609, Oct. 1997.

[21] P. K. Saha, J. K. Udupa, E. F. Conant, D. P. Chakraborty, and D. Sullivan, "Breast tissue density quantification via digitized mammograms," IEEE Trans. Med Imag., vol. 20, no. 8, pp. 792-803, Aug. 2001.
[22] L. Shuqian, L. Xueli, and G. Zhou, "A simplified fuzzy connectedness method used for segmentation of vessel images," in Proc. 25th Annu. Int. Conf. IEEE Eng. Med. Biol. Soc., Sep. 2003, vol. 1, pp. 751-753.

[23] J. Tschirren, E. A. Hoffman, G. McLennan, and M. Sonka, "Intrathoracic airway trees: Segmentation and airway morphology analysis from low-dose CT scans," IEEE Trans. Med. Imag., vol. 24, no. 12, pp. 1529-1539, Dec. 2005.

[24] J. K. Udupa, P. K. Saha, and R. A. Lotufo, "Relative fuzzy connectedness and object definition: Theory, algorithms, and applications in image segmentation," IEEE Trans. Pattern Anal. Mach. Intell., vol. 24, no. 11 , pp. 1485-1500, Nov. 2002.

[25] R. Gonzalez and R. Woods, Digital Image Processing, 2nd ed. Upper Saddle River, NJ: Prentice-Hall, 2002.

[26] J. Dehmeshki, "An adaptive segmentation and 3-D visualisation of the lungs," Pattern Recognit. Lett., vol. 20, pp. 919-926, 1999.

[27] S. Z. Li, Markov Random Field Modeling in Computer Vision. New York: Springer-Verlag, 1995. 